\section{BULLYING EN EL FÚTBOL INFANTIL: LA MIRADA DE LAS FAMILIAS Y DE LOS ENTRENADORES}

\author{
BULLYING NO FUTEBOL INFANTIL: O OLHAR DAS FAMÍLIAS E DOS \\ TREINADORES.
}
BULLYING IN CHILDREN'S FOOTBALL: AN INSIGHT FROM THE FAMILIES' AND COACHES' POINT OF VIEWS

\author{
Maria Prat Grau*, Gonzalo Flores Aguilar**, Xènia Ríos Sisóo**, \\ Carles Ventura Vall-Ilovera***
}

Palabras clave: Bullying.

Fútbol.

Familia.
Entrenador.

\begin{abstract}
Resumen: Este estudio profundiza sobre bullying en el fútbol infantil (8-14 años) dando voz a las familias y los entrenadores de clubs de distintos contextos, características y niveles deportivos. Por ello, se desarrollan grupos focales para que ambos reflexionen sobre los principales factores asociados a su detección, problemática y prevención, a partir de sus experiencias y vivencias personales. Los principales resultados muestran que familias y entrenadores consideran el bullying en el fútbol infantil como un problema importante y necesario de atender. Todos coinciden en afirmar que no disponen de suficiente formación para su detección, ni de estrategias de prevención y tratamiento. Sin embargo, también se constata cierta banalización del problema por parte de los participantes en el estudio. Los resultados permiten identificar los factores que más preocupan a las familias y a los entrenadores, para poder orientar su detección y prevención a partir de los problemas reales planteados.
\end{abstract}

Palavras chave: Bullying

Futebol.

Família.

Treinador.

Keywords:

Bullying.

Soccer.

Family.

Coach.

Resumo: Este estudo investiga o bullying no futebol infantil (8-14 anos), dando voz a famílias e treinadores de clubes de diferentes contextos, características e níveis desportivos. Para este fim, são realizados grupos focais para que ambos reflictam sobre os principais factores associados à sua detecção, problemática e prevenção, com base nas suas experiências e vivências pessoais. Os principais resultados mostram que as famílias e treinadores consideram o bullying no futebol infantil como um problema importante e necessário a abordar. Todos concordam que não têm formação suficiente para detectar ou desenvolver estratégias de prevenção e tratamento. Contudo, observa-se também uma certa banalização do problema por parte de alguns dos participantes no estudo. Os resultados permitem identificar os factores que mais preocupam as famílias e os treinadores, com vista a orientar a sua detecção e prevenção com base nos problemas reais abordados.

Abstract: This study looks further into bullying in children's (aged 8-14) football, listening to families and coaches from different contexts, characteristics and sports levels. Focus groups are developed so that both families and coaches can think about the main factors associated with the detection of bullying, the problems it may cause, and how to prevent it, speaking from their own experiences and personal lives. The findings revealed that families and coaches consider that bullying in children's football is a serious problem that needs to be addressed. They all agree on the fact that they lack information about how to detect, prevent and address bullying. However, participants tend to trivialize the problem somehow. The findings enable us to identify which factors have families and coaches more concerned and can be used to help to detect and prevent bullying based on real problems exposed.
*Universitat Autònoma de Barcelona. Barcelona, España. E-mail:

maria.prat@uab.cat

**Universitat de Vic - Universitat Central de Catalunya. Barcelona, España.

E-mail:

gonzalo.flores@uvic.cat

***Institut Nacional Educació Física de Catalunya. Barcelona, España. E-mail:

xrios@gencat.cat; carlesventura@gencat.cat

Recebido em: 09-10-2019 Aprovado em: 13-04-2020 Publicado em: 04-05-2020 


\section{INTRODUCCIÓN}

El bullying o acoso entre iguales se entiende como un comportamiento de carácter agresivo, con la intención de hacer daño al otro, que se repite durante un tiempo, y que supone una relación interpersonal donde se da un desequilibrio de poder o fuerza (OLWEUS; LIMBER, 2010). Su problemática ha sido ampliamente estudiada desde los años 70, principalmente en el universo escolar, a partir de los trabajos realizados por Olweus en 1978, quien lo clarifica conceptualmente y presenta las primeras investigaciones en Noruega y Suecia.

Teniendo en cuenta que el bullying es una forma de violencia muy concreta, debe diferenciarse de conceptos similares. Algunas revisiones bibliográficas, ya sean en el deporte o en educación física, justifican la necesidad de reconceptualizar y diferenciar lo que es bullying de otros conceptos (BACHAND, 2017; MARTíNEZBAENA; FAUS-BOSCÁ, 2018), ya que a menudo se confunden. Así pues, cabe establecer diferencias entre bullying y harassement puesto que ambos fenómenos tienen similitudes y pueden generar daños, exclusión, intimidación o humiliación. Sin embargo, en el harassement hay una relación de poder establecida desde una posición de autoridad sobre el/la deportista (ejemplo, por parte de un entrenador/a o persona adulta). En cambio, en el bullying la interacción de poder se produce entre iguales. En el caso del deporte entre jugadores/as (STIRLING, 2008). A su vez, bullying y harassement se sitúan como formas concretas de violencia o agresividad en el deporte, concepto que abarca aspectos mucho mas amplios y que se vinculan con la falta de deportividad o fair-play en la práctica deportiva, conductas agresivas respecto al equipo contrario, a los árbitros o al público. Se trata de violencia que implica agresiones físicas, verbales o de otra índole, pero que no necesariamente supone una forma de agresión reiterada entre iguales.

El bullying es un tema relevante que ha sido investigado, sobre todo, en el ámbito escolar, donde se indica que al menos una quinta parte del alumnado se ha percibido como víctima en alguna ocasión. Aún así, un tercio de los episodios de intimidación se producen fuera de este contexto, por ejemplo, en el deporte (ADLER, 2014).

A pesar de la notable presencia de situaciones de bullying en el ámbito deportivo (MATTEY; MCCLOUCHAN; HANRAHAN, 2014; BACHAND, 2017), aún son pocos los estudios sobre el acoso entre iguales en este contexto (EVANS; ADLER; MACDONALD; CÔTÉ, 2016) y la mayoría se han realizado durante los últimos diez años principalmente en Canadá, aunque también en Portugal y el Reino Unido. Además de esta escasez, gran parte de estos trabajos se han focalizado en el análisis de su prevalencia, a partir de indagar sobre su caracterización, tipología, frecuencia, causas, consecuencias, etc., junto al estudio de los perfiles de las víctimas, agresores y espectadores. Muestra de ello, sobresalen los recientes estudios de Nery, 2016; Nery; Neto; Rosado; Smith, 2018; Evans et al., 2016, que nos aproximan de manera descriptiva a conocer la dimensión y alcance del problema, pero aportan poca información sobre la complejidad de este.

Cabe destacar también, que existe una problemática específica en el análisis del bullying en el deporte, ya que en este contexto se asume de forma bastante generalizada como algo inherente a la cultura deportiva, considerando que la 
competición y agresividad forman parte de la esencia del deporte (KERR; JEWETT; MACPHERSON; STIRLING, 2016). Por ello, resulta muy difícil diferenciar entre las situaciones de bullying y las que no, pudiéndose percibir las de bullying como algo normal dentro del ámbito deportivo (FISHER; DZIKUS, 2017). De esta forma, los autores afirman que el bullying en el deporte es un fenómeno importante y muy complejo, ya que los deportistas y entrenadores normalizan un comportamiento abusivo que a su vez constituye un modelo social de aprendizaje. Así pues, para entender estas dinámicas y esta complejidad, se hace necesario utilizar metodologías cualitativas y aproximaciones más globales y comprensivas que nos permitan entender y afrontar el problema de forma integral (SHANNON-MCCALLUM, 2013; KERR et al, 2016; FISHER; DZIKUS, 2017), ya que los estudios cualitativos en el contexto del bullying deportivo son escasos (FISHER; DZIKUS, 2017; NERY, et al. 2018; SHANNON-MCCALLUM, 2013; COLLOT-D’ESCURY; DUDINK, 2010).

El estudio que se presenta se contextualiza en el denominado Modelo Ecológico de Bronfenbrenner (1976) que permite comprender la agresividad entre pares como resultado de procesos de interacción entre variables intrapersonales, interpersonales y grupales, en interacción con el contexto sociocultural donde se desarrollan (PÉREZ, 2011). Este autor, nos describe con detalle las características del modelo. No se trata de poner el foco únicamente en la víctima o el agresor, sino que se necesita explorar y examinar el contexto (ESPELAGE; SWEARER, 2010). Según los autores, gran parte de los estudios lo han analizado como un fenómeno aislado, mientras que debe considerarse como un tándem con el entorno en el que se desarrolla.

Diversas investigaciones consideran que para adoptar políticas de prevención del bullying en el deporte se requiere la implicación de las familias, entrenadores y los clubs deportivos o instituciones (SHANNON-MCCALLUM, 2013; EVANS et al., 2017; NERY et al., 2018). Se trata de un fenómeno de gran complejidad y como tal, debe abordarse de forma amplia y estructural.

Shannon-Mccallum (2013) y Fisher; Dzikus (2017) destacan también la necesidad imperiosa de tener presentes los factores ambientales del propio club o institución deportiva. Las relaciones entre los miembros de la comunidad, las normas y rutinas de funcionamiento, junto a un buen clima social ya que son aspectos que pueden fortalecer la identificación y prevención del problema.

Por tanto, el interés del presente estudio es profundizar en el bullying, concretamente en el fútbol infantil, y en las interacciones con el contexto inmediato en que éste se produce junto con los agentes sociales implicados. Por ello, es fundamental conocer en profundidad qué saben y opinan las familias y entrenadores sobre el tema (ESPELAGE; SWEARER, 2010), qué dudas e inquietudes les genera, qué factores consideran relevantes, y cómo los identifican y valoran a partir de sus propias experiencias y vivencias personales.

\section{METODOLOGÍA}

Esta investigación se ha realizado en base al paradigma cualitativo (PATTON, 2002), ya que la complejidad de esta problemática requiere la necesidad de ser abordarla en profundidad y de manera detallada (TORRANCE, 2000). 
El estudio se desarrolló a partir de un estudio de casos múltiples (YIN, 1989). La selección de los casos participantes (clubs de fútbol infantil) responde a una elección de casos típicos (HEINEMANN, 2003), atendiendo a distintos criterios: (1) clubs con equipos de categorías benjamín (año de nacimiento: 2008 y 2009), alevín (año de nacimiento: 2006 y 2007) e infantil (año de nacimiento: 2005 y 2006); (2) diferentes orígenes geográficos dentro de Cataluña; (3) diferentes entornos (rural/ urbano); y (4) diferentes tamaños de población y clubs.

Una vez seleccionados los clubs participantes se llevaron a cabo un total de seis grupos focales con las familias y los entrenadores que voluntariamente se ofrecieron a participar (grupos entre 10 y 15 personas), todos con perfiles heterogéneos en cuanto al sexo, la categoría y dimensión de los clubs, las edades y los años de experiencia. A pesar de intentar buscar la representatividad de mujeres en los grupos de entrenadores, solo participó una en este colectivo, mientras que, en los grupos de familias, la presencia de mujeres y hombres fue equilibrada. Por otra parte, se pidió la autorización correspondiente al "Comitè d'Ėtica d'Investigacions Clíniques de l'Administració Esportiva de Catalunya" y las personas que voluntariamente participaron, fueron informadas de sus propósitos y de las garantías de confidencialidad y anonimato.

La finalidad de los grupos focales era conocer en profundidad el problema de bullying a partir de la aportación de ejemplos, situaciones y experiencias vividas mediante entrevistas semiestructuradas a un grupo de personas (KRUEGER; CASEY, 2014). En comparación con otras técnicas, los grupos focales son ideales para que surjan actitudes, sentimientos, creencias, experiencias y reacciones profundas entre todos los participantes (ESCOBAR; BONILLA-JIMENEZ, 2009).

Los grupos focales se realizaron en las instalaciones de cada club, o en la escuela de entrenadores, el día y la hora que ellos decidieron. Con la intención de promover la máxima participación a partir de una conversación activa y estimulante, cada grupo focal estuvo moderado por dos personas investigadoras (KRUEGER; CASEY, 2014), las cuales contaron con un guión de preguntas abiertas en base a las temáticas del estudio. Además, todas las conversaciones fueron registradas en audio para posteriormente ser transcritas. En definitiva, todos estos aspectos son destacados en las fases para la elaboración e implementación de los grupos focales de Escobar et al. (2009).

En cuanto a la definición de las categorías de este estudio, en primer lugar, se siguió un proceso deductivo a partir de la fundamentación teórica y, posteriormente, un análisis inductivo de los textos extraídos que permitió redefinir el catalogo inicial, a partir de la incorporación de nuevos temas emergentes y el descarte de los menos representativos y/o relevantes (MILES; HUBERMAN, 1994). Así pues, las dimensiones de este estudio fueron: a) relevancia del problema y/o banalización, b) identificación, invisibilidad y abandono, c) falta de experiencia y formación, d) comunicación y clima de confianza y, e) propuestas de actuación y prevención.

Finalmente, los procesos de explotación, codificación, análisis e interpretación de los resultados se llevaron a cabo siguiendo las fases del análisis de contenido de Bardin (1986). En la fase de "preanálisis y preparación del material" se optó por la 
selección y utilización de todos los datos, sin excepción. En la fase de "explotación del material", las dos personas moderadoras de los grupos focales codificaron abiertamente los resultados con la ayuda del programa informático "Nudist NVivo®". $Y$ en la fase de "tratamiento de resultados e interpretaciones" se generaron y compararon los informes generados por las dos personas investigadoras, los cuales contenían las citas directas de los participantes.

\section{RESULTADOS}

El análisis de los datos obtenidos nos permite identificar y reflexionar sobre los distintos factores que influyen en la problemática del bullying en el fútbol infantil y se exponen agrupados según las dimensiones ya descritas. No se aprecian diferencias relevantes entre las aportaciones de hombres y mujeres participantes, por lo que se presentan las respuestas agrupadas.

\subsection{RELEVANCIA DEL PROBLEMA Y/O BANALIZACIÓN}

Las personas entrevistadas coinciden de forma generalizada en considerar que el bullying es un tema "muy importante" que puede implicar "consecuencias graves para aquellos que lo sufren". Son conscientes que les preocupa y que se trata de algo de "gran complejidad". Lo consideran un problema "delicado y difícil", y que despierta temores e inquietudes, sobre todo a las familias. Algunas de ellas manifiestan "horror" en pensar que alguno de sus hijos pueda ser víctima o agresor, y expresan una gran indefensión ante un problema que les pueda pasar desapercibido.

\footnotetext{
Siempre he pensado que el bullying es una palabra que se oye mucho últimamente. Y algunas veces pienso, ¿y si le pasara a mi hijo? ¿cómo lo haría? Es algo que no sabría qué hacer [...] no sabes cuándo y cómo se genera esta situación (Familia. Caso C).

Un día me enteré de que mi hijo era de los que hacia bullying a un niño, [...] ¡no me lo podía creer! Lo pasamos muy mal (Familia Caso A).
}

Por otra parte, algunos entrenadores y familias lo consideran algo problemático, pero no de forma tan generalizada. Así pues, mientras algunos reconocen que es algo muy importante y requiere de especial atención, algunos lo normalizan o incluso lo banalizan. De esta forma, acciones que pueden ser indicios de bullying pasan a ser calificadas como "una broma", que es "cosa de niños", que "no hay mala intención" o de algo que "forma parte de lo cotidiano", normalizando parte de estas situaciones. "Los insultos y las bromas muchas veces los hacen de forma cotidiana, sin mala fe" (Entrenador. Caso A).

Un tema recurrente es vincular ciertas situaciones de bullying a la naturaleza del deporte. De este modo, surgen expresiones por familias y entrenadores como "el deporte es así", "el fútbol es así", "hay situaciones agresivas", "el deporte es excluyente", "los niños quieren ganar", etc. justificando algunas situaciones potencialmente de bullying, como algo que forma parte del sistema deportivo. Se menciona también la alta competitividad del fútbol infantil, y cómo los entornos del fútbol están "contaminados" por el fútbol que se ve en TV y que los niños deportistas tienden a imitar. 


\subsection{IDENTIFICACIÓN, INVISIBILIDAD Y ABANDONO}

Sumado a lo expuesto, uno de los principales problemas que se aprecia en los debates surgidos es saber discernir entre lo que realmente se trata de bullying de aquello que no lo es, tal y como lo expresan los participantes en numerosas ocasiones:

Es muy difícil para los padres o educadores saber en qué momento se produce el bullying (Familia. Caso B).

He vivido situaciones de burlas, motes, esconder la ropa, etc. Pero no creo que sean situaciones de bullying (Entrenador. Caso A).

A su vez, y lamentablemente, en algunas ocasiones las situaciones de bullying se identifican demasiado tarde, cuando el problema ya es muy grave.

La gente acaba identificando el bullying cuando acaba pasando algo muy grave. Pero que lo importante es que los entrenadores puedan identificarlo en el momento en el que se está produciendo (Entrenador. Caso D).

Se hace necesario estar "atentos a cualquier señal de alarma" y "actuar y cortar rápidamente cualquier indicio de agresión: Tolerancia 0”.

Los agresores actúan cuando saben que no serán vistos y en gran parte de las ocasiones se produce sin la presencia de los adultos. Por ello, su difícil detección y la invisibilidad del problema se conciben como los principales obstáculos de este.

\footnotetext{
El tema de detectarlo fue muy difícil porque siempre se buscaba el momento y las horas que los entrenadores no estábamos presentes. Momentos de la ducha, por ejemplo (Entrenador. Caso E).

Yo creo que los niños no son tontos y saben cuando hacer bullying o no [...] Cuando alguien quiere hacer daño, actúa cuando el entrenador no está (Entrenador. Caso E).
}

Por otra parte, los mismos entrenadores indican que el bullying físico lo puedes detectar enseguida, mientras que el bullying psicológico es más sutil y difícil de detectar.

Bueno yo no sabía nada porque el chaval aparentemente parecía feliz, pero un día me escribió un whatsapp; me dijo que estaba hundido y lo estaba pasando fatal. Yo no me lo esperaba porque es un chaval que normalmente siempre está feliz y sonriendo. (Entrenador. Caso D).

Una de las principales conclusiones que se desprende de las discusiones es considerar que posiblemente parte de los niños o niñas que sufren bullying en los centros deportivos abandonan el club. Por ello, quizás encontremos menos presencia de bullying en los mismos que en el contexto escolar.

\footnotetext{
Fue tal el bullying que sufrieron esos tres jugadores que ambos se pusieron de acuerdo y decidieron marcharse. Estaban amargados, no querían jugar, no querían estar en el equipo (Entrenador. Caso D)

Mi hijo mayor sufrió bullying en este club y finalmente abandono el fútbol y se marchó (Familia. Caso B).
}

Pertenecer a un club pequeño puede facilitar su detección, ya que en un club pequeño todas las familias se conocen, los entrenadores son más cercanos y accesibles, y la comunicación suele ser directa, etc. En cambio, en un club grande el problema puede pasar desapercibido. 
Yo pienso que en este club no es tan complicado porque se puede hablar con los padres y los entrenadores también son próximos. Claro, es un club pequeño (Familia. Caso $A$ ).

En el caso del club rural, los participantes manifiestan que lo que acontece en la escuela se traspasa al club y viceversa. En el caso de los clubs pequeños, se sugiere la posibilidad de establecer lazos con la escuela para la prevención del problema.

\subsection{FALTA DE EXPERIENCIA Y FORMACIÓN}

Entre los resultados surge de forma recurrente la falta de experiencia y de formación de los entrenadores, ya que en su gran mayoría suelen ser muy jóvenes y con poca formación. En más de una ocasión se reconoce que no están preparados para poder afrontar situaciones de bullying y conflictos parecidos.

\footnotetext{
Son chicos muy majos y tienen muchos conocimientos de fútbol, pero son muy jóvenes y hay cosas que se aprenden con el tiempo y con la experiencia. (Familia. Caso A).

La mayoría de los entrenadores saben de fútbol y de técnicas deportivas, pero poco de pedagogía, y no disponen de herramientas para afrontar los conflictos (Entrenador. Caso E).
}

De esta forma, los propios entrenadores son conscientes de sus limitaciones, y algunos manifiestan que en el fútbol infantil "es más importante ser un buen educador que un buen entrenador" (Entrenador. Caso E).

La formación constituye pues uno de los factores más importante para la prevención del problema. De hecho, todos los grupos de participantes (entrenadores y familias) exponen no haber realizado ningún tipo de formación específica sobre el bullying. Aún así, la necesidad de formación se propone básicamente a tres niveles: a) formación dirigida a las familias para saber cómo deben actuar, b) formación de los monitores como máximos responsables directos que están en contacto diario con los niños y c) formación dirigida a los deportistas. "La Federación debería dar charlas, conferencias, talleres, a los niños, padres, entrenadores y coordinadores del club" (Entrenador. Caso E).

Entrenadores y familias reclaman la necesidad de disponer de estrategias para afrontar el problema y admiten no conocer ni disponer protocolos específicos de actuación en caso de producirse situaciones de bullying. De hecho, existe una gran inseguridad y muchas dudas sobre cómo intervenir en este tipo de conflictos.

\subsection{COMUNICACIÓN Y CLIMA DE CONFIANZA}

La necesidad de establecer una buena comunicación entre familias, entrenadores y los clubs ha sido otro tema destacado. De ahí salen propuestas como realizar reuniones antes y durante la temporada, búsqueda de proximidad entre estos colectivos y articular canales para facilitar intervenciones. A pesar de ello, y de forma minoritaria, algunos entrenadores prefieren no hablar con las familias y mantener cierta distancia con los mismas.

Este año hice una primera reunión con las familias. Creo que la comunicación entre el entrenador y las familias es vital (Entrenador. Caso E). 
Personalmente, como entrenador, no me gusta hablar con las familias porque creo que al final se puede llegar a un nivel de excesiva confianza que tienes que dar explicaciones de todo (Entrenador. Caso E).

Según las aportaciones de las familias, también se requiere de una buena comunicación entre padres e hijos, acompañada de una confianza mutua, ya que ésta es esencial para detectar el conflicto de forma rápida y buscar soluciones. De esta forma, las familias consideran que la prevención empieza a partir de una buena comunicación que facilite la detección del problema y permita actuar. Sin embargo, algunas familias lamentan que en determinadas edades algunos niños "son muy cerrados, son una tumba y no explican nada"; aunque esto no siempre es así. "La gran ventaja es que el niño se lo dijo a sus padres y ellos hablaron con los entrenadores. Si el crío se cierra y nadie se entera" (Familia. Caso B).

Si el clima de confianza entre familias e hijos es valorado como fundamental, también lo debe ser entre niños y entrenadores. El entrenador comparte más horas con los deportistas y es quien debe intervenir en primera instancia.

Creo que es importante tener confianza con el entrenador. En mi caso, un niño que tenia un problema vino a explicármelo y yo hablé con su padre. Este me lo agradeció y me dijo que a partir de nuestra conversación las cosas mejoraron (Entrenador. Caso E).

Además, el entrenador suele ser un referente para los niños con quien establecen lazos de amistad y confidencialidad. Ante situaciones conflictivas, puede resultar más fácil la comunicación con ellos que con sus familias.

\footnotetext{
Si tu eres su amigo y el chico sufre mucho, te lo dirá. Antes te lo dirá a ti que su propia familia (Entrenador. Caso A).

Tu eres un referente para los chicos, porque sabes que pasa, como es su rendimiento, su estado anímico, etc. Los chicos te admiran, valoran tu trabajo y te buscaran a ti (Entrenador. Caso E).
}

También las familias asumen parte de su responsabilidad en situaciones de bullying a través de su propio comportamiento. Haciendo autocrítica afirman: "Todos los padres llevan un entrenador dentro [...] todos los padres saben mucho de fútbol", y admiten que no siempre tienen un comportamiento adecuado.

El rol de las familias es muy importante creo yo. En los partidos, dices: "no se la pases a aquel" [...] "al patoso no", [...] en cambio, animar nos cuesta mucho más. De manera inconsciente los adultos intervenimos y los niños lo captan (Familia. Caso A).

De este modo, se pone de manifiesto que la actitud de padres y madres, y sus intervenciones pueden ser determinantes para reforzar o inhibir determinados conflictos.

\subsection{PROPUESTAS DE ACTUACIÓN Y PREVENCIÓN}

Algunas iniciativas y propuestas concretas orientadas al club surgidas en los debates son: a) la necesidad de articular protocolos de actuación en caso de detección del bullying: concretar a quién acudir, de qué manera, qué se debe hacer y qué no, etc.; b) diseñar campañas de sensibilización y educación en valores de forma continuada, como ya hace uno de los clubs; c) disponer de algún tipo de ayuda externa, 
de un psicólogo o asesor externo, etc. a pesar de la falta de presupuesto para ello; y d) mantener contacto y comunicación de forma periódica entre los entrenadores, las familias (reuniones, celebraciones, etc.) y los jugadores, más allá de lo estrictamente deportivo, además de promover la creación de espacios para tal fin.

Todos los participantes coinciden en subrayar la prevención por parte del club, las familias y los entrenadores, como eje clave para combatir el bullying. Según las ideas aportadas por los distintos grupos, ante la presencia de los primeros síntomas de bullying es necesario actuar de forma inmediata con "firmeza, responsabilidad y sensibilidad" y no esperar que el problema se haga mayor y se escape de control.

[Ante un caso de bullying]. Yo sé que la familia lo comentó dos o tres veces al club. Si el club hubiera actuado la primera vez, no se hubiera alargado la cosa. Cuando nos enteramos era ya tarde [...] Cuando hicieron algo era ya la tercera vez que el padre fue a quejarse. Aquí el club no actuó correctamente (Entrenadores. Caso D).

Familias y entrenadores coinciden en actuar conjuntamente y de manera contundente, junto con la complicidad del club y los máximos responsables del mismo.

\section{DISCUSIÓN}

La revisión de la literatura y los resultados obtenidos aportados por familias y entrenadores nos permiten analizar, contrastar y profundizar sobre los principales factores que inciden en la identificación y prevención del bullying en el fútbol infantil.

Un primer factor destacado es conocer el grado de preocupación de las familias y entrenadores, y aquí se han desvelado dos posicionamientos claros: por un lado, de una forma casi unánime, todas las familias y entrenadores, lo consideran como un problema de primer orden y de extrema gravedad. En cambio, existe una segunda postura, sobre todo adoptada por parte de algunos entrenadores, en la que se banaliza el problema, hasta el punto de considerarlo como algo normal y propio de la naturaleza competitiva del mismo deporte. Esta ambivalencia coincide con estudios previos realizados (SHANNON-MCCALLUM, 2013; KERR et al. 2016; MATTEY et al. 2014; FISHER y DZIKUS, 2017; NERY et al, 2018). Además, familias y entrenadores advierten del daño y sufrimiento que puede causar el fenómeno, pero a su vez, una parte del colectivo entrevistado desvela la normalización o banalización del problema.

En los entornos deportivos, la banalización del conflicto puede resultar mayor que en otros contextos. Así pues, se puede llegar a generar un clima de tolerancia que promueva e incluso refuerce los comportamientos agresivos (SHANNONMCCALLUM, 2013). En esta línea, los estudios de Fisher y Dzikus (2017) utilizan el término de "overconfirmity" como parte del sistema deportivo, según el cual se asume, en cierta medida, que la práctica deportiva va asociada con la aceptación del sufrimiento, la superación de obstáculos, y permitir las típicas novatadas o bromas para ser aceptado por el equipo (COAKLEY, 2016; VVEINHARDT, et al. 2019). Esta mirada de la problemática, forma parte de la banalización del problema surgido también en los resultados del estudio.

Saber identificar y diferenciar lo que es bullying de lo que no lo es, se considera otro aspecto de vital importancia (VVEINHARDT et al. 2019). En consonancia 
con los resultados obtenidos, Mishna, et al. (2006), describen el bullying como un tema confuso y de gran complejidad, en el que las familias y educadores no saben distinguir entre aquello que es normal de lo que no lo es. Como consecuencia de ello, surgen situaciones de inseguridad y no se sabe cómo intervenir. Para aproximarse a un problema es necesario saber identificarlo y reconocerlo. Por esto, para ofrecer respuestas efectivas se precisa la clarificación del concepto y el análisis de los factores que intervienen (BACHAND, 2017). De hecho, los resultados del estudio coinciden con estas aportaciones, ya que hemos constatado también como entrenadores y familias expresan de forma reiterada problemas y dudas para saber reconocerlo e identificarlo.

Otro rasgo característico que se desprende de los resultados obtenidos es la invisibilidad del problema -aspecto ya advertido en investigaciones previas. Atendiendo a las diversas tipologías del bullying y a las condiciones en que este se produce, el bullying físico es fácilmente detectable, mientras que el bullying social o psicológico, que se manifiesta de forma más sutil, resulta mucho más peligroso puesto que da en contextos donde no hay presencia del adulto (los vestuarios son los espacios más frecuentes) o fuera del club. (NERY et al., 2018).

Junto al problema anterior, Nery et al. (2018), entre otros, se refieren a la denominada "ley del silencio" que suele acompañar gran parte de los episodios de bullying. De esta forma, si el problema no se comunica, parece que no existe. En el caso del deporte, al tratarse de una actividad voluntaria, la "ley del silencio" incluso puede quedar anulada porque algunas situaciones de bullying acaban derivando hacia el abandono o retirada de los deportistas afectados (VVEINHARDT, et al. 2019). En este estudio se han puesto en evidencia casos destacados de abandono del fútbol cuando las víctimas no encontraban otras soluciones alternativas para superar el problema. En esta línea, las aportaciones de Shannon-McCallum (2013) muestran como las situaciones de abandono perjudican gravemente a las víctimas, ya que les priva de la oportunidad de participar en las prácticas deportivas y beneficiarse de los aspectos positivos de las mismas, además del sufrimiento que se les genera.

Coincidiendo con la literatura, la presencia del bullying en la escuela es mucho más frecuente que en el contexto deportivo (NERY et al. 2018; EVANS et al., 2016). Los autores confirman la tendencia de las víctimas en abandonar el club cuando lo sufren. Bachand (2017), señala que en los dos contextos el bullying está presente pero que son ámbitos difícilmente comparables. Sin embargo, todavía son pocos los estudios que comparen el fenómeno del bullying en las escuelas y en los entornos deportivos (VVEINHARDT et al. 2019).

Entrenadores y familias han identificado la comunicación como otro de los factores importantes para abordar la problemática; una idea esencial para la prevención en las organizaciones deportivas (SHANNON-MCCALLUM, 2013). En su estudio, la autora insiste en la falta de sensibilidad y de mecanismos de las organizaciones deportivas para proponer códigos de conducta y establecer canales de comunicación con los responsables del programa para afrontar el problema.

Por otra parte, fortalecer la relación entrenador-deportista puede considerarse como una de las piezas más importantes y potencialmente influyentes para la prevención, ya que los entrenadores ejercen un gran poder sobre los deportistas. En 
el estudio realizado, los entrenadores son plenamente conscientes de su influencia sobre los niños y niñas, ya que admiten "ser un referente". En ocasiones, incluso se consideran "un amigo" de los mismos, y afirman que posiblemente confíen antes con ellos que en sus familias. Bachand (2017), apunta que el tiempo compartido entre entrenadores y deportistas puede ejercer mucha presión y tolerar acciones que normalmente serian inaceptables. En esta línea, se reclama una actitud firme y comprometida de los educadores frente al problema (MENESINI; SALMIVALLI, 2017). Para ello, resulta sumamente importante que los entrenadores expresen de forma clara su actitud anti-bullying hacia los deportistas; esto es lo que también se ha descrito como tolerancia 0 (ROBERGE, 2012). De esta forma, las actitudes de los entrenadores se configuran como factores esenciales para intervenir de forma efectiva en estos contextos.

La formación se ha identificado también como un factor fundamental para la prevención del bullying en el fútbol infantil, sobre todo por las grandes carencias de los entrenadores para dar una respuesta adecuada y efectiva al conflicto (SHANNONMCCALLUM, 2013; VVEINHARDT et al. 2019). En este sentido, Shannon-McCallum (2013) concluye que el personal debe ser formado sobre el bullying: qué es, cómo reconocerlo, cómo actuar para prevenirlo y qué procedimientos deben seguir en caso de que se produzca la intimidación. Por ello, es importante que los entrenadores no dominen únicamente técnicas y tácticas deportivas, sino que adquieran competencias sociales que impliquen estrategias de prevención e intervención en situaciones de vulnerabilidad (COLLOT-D’ESCURY; DUDINK, 2010).

Existe unanimidad por parte de los participantes en el estudio en abordar la problemática desde los distintos agentes sociales, y especialmente desde el ambiente del club o institución. Fisher; Dzikus (2017) lo denominan "atmósfera moral" o "clima deportivo inclusivo". Se trata de que las organizaciones deportivas faciliten y garanticen la creación de "entornos de seguridad y bienestar" siendo objetivos urgentes para formularse desde las mismas (VVEINHARDT et al. 2019).

Los estudios de Menesini; Salmivalli (2017); Mishna, et al. (2019), sugieren incorporar padres y madres en los programas formativos y establecer propuestas de actuación conjunta con la comunidad educativa junto con la sensibilización del alumnado (deportistas en el caso que nos ocupa). Este aspecto se considera fundamental ya que hemos constatado la existencia de familias que, aunque no hayan sufrido nunca bullying directamente, se podrían considerar posibles víctimas. En el ámbito deportivo, se dispone de escasa de literatura sobre cómo debe ser la implicación de las familias (VVEINHARDT et al. 2019).

Las propuestas de actuación y de intervención se concretan en todos los niveles, y por ello se necesita conocer la perspectiva de los estudiantes (deportistas), familias y educadores (entrenadores) (SHANNON-MCCALLUM, 2013; MISHNA et al. 2006). Se requiere una intervención enérgica por parte de los entrenadores, de los responsables de las instituciones y de las familias (ORNELAS; OLIVEIRA, 2013). En sentido contrario, a pesar de ser conscientes de la falta de formación, algunos clubs no se muestran particularmente interesados en organizar cursos de formación y prevención del problema (VVEINHARDT et al. 2019). 
Finalmente, cabe destacar que la práctica deportiva bien desarrollada puede llegar a ser incluso un factor protector ante posibles situaciones de bullying, puesto que aporta confianza y empodera a los practicantes (ORNELAS; OLIVEIRA, 2013). Sin embargo, los autores admiten que puede ser también un factor de riesgo si no se desarrolla de forma adecuada.

Así pues, del presente estudio se desprende que el deporte no es necesariamente un factor protector contra el bullying. Al contrario, participar en deporte, y en el caso estudiado en el fútbol infantil, puede ser un contexto de riesgo con respecto a la vulnerabilidad hacia comportamientos de agresión o intimidación (COLLOT-D’ESCURY; DUDINK, 2010). De hecho, el exceso de competitividad, típico en la cultura del fútbol infantil, puede incrementar las situaciones de bullying.

\section{CONCLUSIONES}

Este estudio contribuye al conocimiento sobre el problema del bullying en el fútbol infantil y aporta un análisis en profundidad sobre los aspectos que más preocupan a las familias y a los entrenadores sobre el problema y su prevención.

Los factores identificados cómo más relevantes del bullying en el fútbol infantil son diversos. Se constatan divergencias en cuanto a la percepción del problema, ya que mientras unos lo consideran como un problema muy grave, otros pueden banalizarlo y considerarlo como parte de la propia "cultura deportiva"; sin embargo, existe unanimidad en considerar el bullying cómo un problema muy complejo y de difícil detección. Como consecuencia, resulta difícil saber cómo y cuando intervenir debido a su invisibilidad, como parte de la propia naturaleza del bullying.

A diferencia del bullying escolar, el bullying en el fútbol infantil, al tratarse de una actividad no obligatoria, puede que las víctimas que lo sufran abandonen o cambien de club sin que se visualice el conclicto. Si no se visualiza el problema, parece que éste no existe, pero el estudio demuestra que no siempre es así, ya que se han identificado diversas situaciones y ejemplos de intimidación.

El ambiente o "atmósfera moral" del club, los canales de comunicación y la relación entre entrenadores, familias y jugadores, son elementos clave para su detección y prevención. Ante la presencia de cualquier indicio de bullying, se propone una reacción enérgica y contundente por parte de todos los agentes implicados: tolerancia 0 .

Finalmente, se pone de manifiesto una carencia de formación específica por parte de las familias, entrenadores, responsables de clubs y jugadores, aspecto considerado necesario y fundamental para abordar su prevención. Por ello, se apela a la necesidad de diseñar e implementar programas formativos dirigidos a todos los agentes sociales implicados. 


\section{REFERENCIAS}

ADLER, Lauren. An examination into bullying in the adolscent sport context.. Tesis (doctoral) - School of Kinesiology and Health Studies. Queen's University. Kingston, Ontario, 2014. Disponible en: https://qspace.library. queensu.ca/bitstream/handle/1974/12496/Adler_Adler_L_201409_MSc. pdf;jsessionid=50034A457837E7B8E5B6A6B2E378214D? sequence=1. Acceso en: 6 jul. 2019.

BACHAND, Charles. Bullying in Sports: The Definition Depends on Who You Ask. Sport Journal, v.21, n.1, 2017. Disponible en: https://thesportjournal.org/article/bullying-in-sportsthe-definition-depends-on-who-you-ask/. Acceso en: 5 jul. 2019.

BARDIN, Laurence. El análisis de contenido. Madrid: Akal, 1986.

BRONFENBRENNER, Urie. The ecology of human development: history and perspectives. Psychologia, v. 19, n. 5, p.537-549, 1976.

COAKLEY, Jay. Sports in society: Issues and controversies. $12^{\text {th }}$ ed. Boston: McGraw, 2016.

COLLOT-D'ESCURY, Annematt; DUNDIK AD. Bullying Beyond School. In: JIMERSON, Shane; SWEARER, Susan; SPELAGE, Dorothy. (Eds.). Handbook of bullying in schools: An international perspective. New York, NY: Taylor \& Francis. Routledge, 2010. p.235248.

ESCOBAR, Jazmine; BONILLA-JIMENEZ, Francy I. Grupos focales: una guía conceptual y metodólogica. Cuadernos Hispanoamericanos de Psicología, v.9, n.1, p.51-67, 2009.

ESPELAGE, Dorothy; SWEARER, Susan. A social-Ecological Model for Bullying Prevention and Intervention. Understanding the impact of Adults in the Social Ecology of Youngsters. In: JIMERSON, Shane; SWEARER, Susan; SPELAGE, Dorothy. (Eds.). Handbook of bullying in schools: an international perspective. New York: Taylor \& Francis Routledge, 2010. p.6172.

EVANS, Blair; ADLER, Ashler; MACDONALD, Dany; CÔTÉ, Jean. Bullying Victimization and Perpetration among Adolescent Sport Teammates. Pediatric Exercise Science, v. 28, n.2, p. 296-303, 2016. DOI 10.1123/pes.2015-0088 Disponible en: https://journals. humankinetics.com/view/journals/pes/28/2/article-p296.xml. Acceso en: 22 jul. 2019.

FISHER, Leslee A.; DZIKUS, Lars. Bullying in Sport and Performance Psychology. Oxford Research Encyclopedia of Psychology, v.1, p.1-23, 2017. DOI 10.1093/ acrefore/9780190236557.013.169. Dispobible en: https://oxfordre.com/psychology/ view/10.1093/acrefore/9780190236557.001.0001/acrefore-9780190236557-e-169. Acceso en: 18 jul. 2019.

HEINEMANN, Klaus. Introducción a la metodología de la investigación empírica en las ciencias del deporte. Barcelona: Paidotribo, 2003.

KERR, Gretchen; JEWETT, Rachel; MACPERSON, Ellen; STIRLING, Ashley. Student-Athletes' Experiences of Bullying on Intercollegiate Teams. Journal for the Study of Sports and Athletes in Education, v.10, n. 2, p.132-149, 2016. DOI 10.1080/19357397.2016.1218648._Disponible en: https://www.tandfonline.com/doi/full/10.10 80/19357397.2016.1218648. Acceso en: 10 sept. 2019.

KRUEGER, Richard A.; CASEY, Mary A. Focus groups: A practical guide for applied research. Washington, DC: Sage, 2014. 
MARTÍNEZ-BAENA, Alejandro; FAUS-BOSCÁ, Joan. Acoso escolar y Educación Física: una revisión sistemática. School Bullying and Physical Education: A systematic review. Retos, v. 34, n. 2, p. 412-419, 2018. Disponible en: https://recyt.fecyt.es/index.php/retos/article/ view/59527. Acceso en: 10 sept. 2019.

MATTEY, Emma; MCCOUGHAN, Lana J.; HANRAHAN, Stephanie. J. Anti-Vilification Programs in Adolescent Sport. Journal of Sport Psychology in Action, v.5, n. 3, p.135146, 2014. DOI 10.1080/21520704.2014.925528. Disponible en: https://www.tandfonline. com/doi/abs/10.1080/21520704.2014.925528. Acceso en: 11 sept. 2019.

MENESINI, Ersilia; SALMIVALLI, Christina, 2017. Bullying in schools: the state of knowledge and effective interventions. Psychology, Health \& Medicine, v.22, n. S1, p.240-253, 2017. DOI 10.1080/13548506.2017.1279740.

MILES, Matthew B.; HUBERMAN, Michael A. Qualitative data analysis: an expanded sourcebook. $2^{\text {nd }}$. ed.. Thousand Oaks, California: Sage. 1994.

MISHNA, Faye; PEPLER, Debra; WIENER, Judith. Factors associated with perceptions and Responses to Bullying Situations by Children, Parents, Teachers and Principals.

Victims \& Offenders, v.1, p.255-288, 2006. DOI 10.1080/15564880600626163.

Disponible en: https://www.tandfonline.com/doi/abs/10.1080/15564880600626163. Acceso en: 10 sept. 2019.

MISHNA, Faye; KERR, Gretchen; MACINROY, Lauren B. MCPERSON, Ellen. Student athletes ' experiences of bullying in intercollegiate sport. Journal for the Study of Sports and Athletes in Education, v.13, n.1, p. 53-73, 2019. DOI 10.1080/19357397.2019.1581512

NERY, Miguel. Bullying no contexto da formação desportiva em Portugal: estudo explorarório a nível nacional de modalidades individuais, colectivas e de combate. Tesis (Doctor en Motricidad Humana, especialidade de Comportamento Motor) - Faculdade de Motricida Humana.Universidade de Lisboa, Lisboa, 2016. Disponible en: https://www. repository.utl.pt/handle/10400.5/13034. Acceso en: 3 jul. 2019.

NERY, Miguel; NETO, Carlos; ROSADO, Antonio; SMITH, Peter. Bullying in youth sport training: a nationwide exploratory and descriptive research in Portugal. European Journal of Developmental Psychology, n. 5629, p. 1-17, 2018. DOI 10.1080/17405629.2018.1447459

OLWEUS, Dan; LIMBER, Susan. The Olweus Bullying Prevention Program. Implementation and evaluation over two Decades. In: JIMERSON, Shane; SWEARER, Susan; SPELAGE, Dorothy. (Eds.). Handbook of bullying in schools: an international perspective. New York: Taylor \& Francis Routledge, 2010. p. 377-40.

ORNELAS, Fernando M.; OLIVEIRA, Beatriz. Prática desportiva, um meio de prevenção do bullying na escola? Movimento, v.19, n.2, p. 55-77, 2013. DOI 10.22456/19828918.30119 Disponible en: https://seer.ufrgs.br/Movimento/article/view/30119. Acceso en: 10 sept. 2019.

PATTON, Michael Q. Qualitative research and evaluation methods. $3^{\text {rd }}$.ed. Thousand Oaks, CA: Sage, 2002.

PÉREZ, Verónica. Percepción de gravedad, empatia y disposición a intervenir en situaciones de bullying físico, verbal y relacional en profesores de $5^{\circ}$ a $8^{\circ}$ básico. Psykhe, v.20, n.2, p.25-37, 2011. DOI 10.4067/S0718-22282011000200003. Disponible en: http:// www.psykhe.cl/index.php/psykhe/article/view/00003. Acceso en: 1 sept. 2019. 
ROBERGE, Ginette. From zero tolerance to early inervention: the evolution of school anti-bullying policy. Ejournal of Education Policy, 2012. Disponible en: https://www. semanticscholar.org/paper/From-Zero-Tolerance-to-Early-Intervention\%3A-The-of-Roberge 198113f8cd1f18663417e03a9d00e0b2d28d1a609. Acceso en: 25 jul. 2019.

SHANNON-MCCALLUM, Charlene S. Bullying in Recreation and Sport Settings: Exploring Risk Factors, Prevention Efforts, and Intervention Strategies. Journal of Park and Recreation Administration, v.31, n.1, p.15-33, 2013. Disponible en: https://js.sagamorepub. com/jpra/article/view/2711. Acceso en: 9 jul. 2019.

STIRLING, Ashley. Definition and constituents of maltreatment in sport: Establishing a conceptual framework for research practitioners. British Journal of Sports Medicine, v.43, n.14, p.1091-1099, 2008. DOI 10.1136/bjsm.2008.051433.

TORRANCE, Deirdre. Qualitative studies into bullying within special schools. British Journal of Special Education, v.27, p.16-21, 2000. DOI 10.1111/1467-8527.t01-1-00151.

VVEINHARDT, Jolita; FOMINIENE, Vilija; ANDRIUKAITIENE, Regina. "Omerta" in Organised Sport: Bullying and Harassment as Determinants of Threats of Social Sustainability at the Individual Level. Sustainability, v.11, 2019. DOI 10.3390/su11092474.

YIN, Robert. Case Study Research: Design and Methods, Applied Social Research Methods Series. London: Sage, 1989.

\section{Apoio:}

Este trabajo forma parte del proyecto "Estudi sobre l'assetjament entre iguals (bullying) a l'àmbit esportiu -fútbol- a Catalunya"; (PARINEFC 0003, 2019). Subvencionado por la Fundación Barça. 